\title{
Vitamin-D and Cardiac Dysfunction in Parkinson's disease: Beginning to Close the Evident Gaps
}

\author{
Fulvio A. Scorza ${ }^{1}$, Renato Leça ${ }^{2}$, Carla A. Scorza ${ }^{1}$, Fernando L.A. Fonseca ${ }^{2}$, Efraín Olszewer ${ }^{3}$, Josef \\ Finsterer $^{4 *}$ \\ ${ }^{\mathrm{T}}$ Disciplina de Neurociência Universidade Federal de São Paulo/Escola Paulista de Medicina (UNIFESP/EPM) São \\ Paulo, Brasil. \\ ${ }^{2}$ Faculdade de Medicina do ABC, Santo André, SP, Brasil. \\ ${ }^{3}$ Fundação de Apoio à Pesquisa e Estudos na Área de Saúde -FAPES \\ ${ }^{4}$ Krankenanstalt Rudolfstiftung, Vienna, Austria \\ *Corresponding Author \\ Josef Finsterer, MD, PhD
}

Article History

Received: 27.01.2020

Accepted: 04.02.2020

Published: 09.02.2020

Keywords: Parkinson's disease (PD), neurodegenerative disorders, malignant disease, SUDPAR.

\section{LETTER TO THE EDITOR}

We read with great enthusiasm the opinion paper by Kim et al. who postulated appropriately that vitamin-D plays a role in the pathogenesis of olfactory dysfunction in Parkinson's disease (PD) [1].

$\mathrm{PD}$, one of the most frequent age-related neurodegenerative disorders, affects millions of people globally, there is no cure, and its prevalence will double by 2030 [2]. In addition to these perspectives, a complicating issue not frequently discussed in the neuroscientific community is the mortality in PD [3]. Unfortunately, PD can be considered a malignant disease because it is associated with higher mortality rates compared to the general population [3, 4]. Clearly, the main causes of death in PD are pneumonia, cerebrovascular and cardiovascular diseases [5]. Additionally, sudden unexpected death in PD (SUDPAR) is increasingly discussed as a contribution to mortality in DP [3, 6].

Didatically, SUDPAR is defined as unexpected death of a patient with PD without any satisfactory cause of death as determined by autopsy [3,6]. Until now, causes of SUDPAR remain elusive [3,6]. Anyway, the results of previous experimental and clinical studies suggest that cardiac abnormalities and autonomic dysfunction play key roles in SUDPAR [6]. Furthermore, a number of risk factors may be directly associated with SUDPAR such as age at onset, duration of PD, gender, motor severity and drug treatment (polypharmacy) [3,6], but these factors require further investigations in translational studies. In addition, it is well discussed that low vitamin-D levels and PD are considered bad fellows [7]. In these lines, systematic reviews and meta-analyses of observational studies have shown that low vitamin D levels are more prevalent in PD patients compared to healthy individuals, and low serum or plasma vitamin-D may be a predictive marker for risk and severity of PD [8].

Despite all these proposals, important studies in the last two decades have shown that vitamin-D is involved in the regulation of the cardiovascular system. In this way, a growing body of data clearly indicate that vitamin-D deficiency is related in the genesis of coronary heart disease, cardiovascular dysfunction, and hence sudden cardiac death $[9,10,12]$. Thus, it has been determined that vitamin-D deficiency is associated with arterial hypertension, left ventricular hypertrophy, congestive heart failure, and chronic vascular inflammation [9-12].

Copyright @ 2020: This is an open-access article distributed under the terms of the Creative Commons Attribution license which permits unrestricted use, distribution, and reproduction in any medium for non commercial use (NonCommercial, or CC-BY-NC) provided the original author and source are credited. 
Based on clinical evidence that clearly points to vitamin-D-mediated cardioprotection, is it possible that vitamin-D concentrations can be considered an interesting biological indicator for possible cardiac abnormalities in PD patients and even SUDPAR? In the near future, probably yes.

In a study of 145 PD patients serum vitamin-D levels were significantly lower as compared to age-matched controls [13]. This may have implications in terms of bone health and fracture risk [13]. This study also provided evidence that the outcome of PD patients with vitamin-D deficiency is worse as compared to those with normal vitamin$\mathrm{D}$ values. Thus, vitamin-D serum levels should be determining din all PD patients and supplementation initiated. However, there are also studies, which did not show a significant role of vitamin-D in the pathogenesis of PD [14].

Overall, there is a long way to go. First, more well-delineated experimental and clinical studies are needed to establish the effect of vitamin-D on PD. As previously has been suggested, human neuroprotection studies are needed, but probably not feasible until better biomarkers are established [7]. Second, we must be attentive and improve impaired cardiovascular functions in PD. Finally, we will also have to explore the specific mechanisms of action that vitamin-D plays, in order to improve cardiac function and to prevent the possible cardiac abnormalities present in patients with PD.

\section{ACKNOWLEDGEMENTS}

Our studies are supported by the following grants: FAPESP (Fundação de Amparo à Pesquisa do Estado de São Paulo); CNPq (Conselho Nacional de Desenvolvimento Científico e Tecnológico); Coordenação de Aperfeiçoamento de Pessoal de Nível Superior (CAPES), AFIP (Associação Fundo de Incentivo à Pesquisa) and FAPESP/CNPq/MCT (Instituto Nacional de Neurociência Translacional).

\section{REFERENCES}

1. Kim, J.E., Oh, E., Park, J., Youn, J., Kim, J.S., Jang, W.(2018). Serum 25-hydroxyvitamin D3 level may be associated with olfactory dysfunction in de novo Parkinson's disease. J Clin Neurosci. 2018 (in press)..

2. Soukup, S.F., Vanhauwaert, R., Verstreken, P. (2018).Parkinson's disease: convergence on synaptic homeostasis. EMBO J.

3. Scorza, F.A., do, Carmo, A.C., Fiorini, A.C., Nejm, M.B., Scorza, C.A., Finsterer, J., Ferraz, H.B.(2017). Sudden unexpected death in Parkinson's disease (SUDPAR): a review of mpublications since the decade of the brain. Clinics. 72:649-651.

4. Poewe, W., Seppi, K., Tanner, C.M., Halliday, G.M., Brundin, P., Volkmann, J. (2017). Parkinson disease. Nat Rev Dis Primers, 3:17013.

5. Pinter, B., Diem-Zangerl, A., Wenning, G.K., Scherfler, C., Oberaigner, W., Seppi, K.(2015). Mortality in Parkinson's disease: a 38-year follow-up study. Mov Disord. 30:266-269.

6. Scorza, F.A., Fiorini, A.C., Scorza, C.A., Finsterer, J.(2018). Cardiac abnormalities in Parkinson's disease and Parkinsonism. J Clin Neurosci, 53:1-5.

7. Peterson, A.L. (2014). A review of vitamin D and Parkinson's disease. Maturitas; 78:40-4.

8. Koduah, P., Paul, F., Dörr, J.M. (2017). Vitamin D in the prevention, prediction and treatment of neurodegenerative and neuroinflammatory diseases. EPMA $J, 8: 313-325$.

9. Lee, J.H., O'Keefe, J.H., Bell, D., Hensrud, D.D., Holick, M.F. (2008). Vitamin D deficiency an important, common, and easily treatable cardiovascular risk factor? J Am Coll Cardiol, 52:b1949-1956.

10. Zittermann, A. (2006). Vitamin D and disease prevention with special reference to cardiovascular disease. Prog Biophys Mol Biol, 92: 39-48.

11. Holick, M.F. (2007). Vitamin D deficiency. N Engl J Med, 357: 266-281.

12. Scorza, F.A., Albuquerque, M.D., Arida, R.M., Terra, V.C., Machado, H.R., Cavalheiro, E.A.(2010). Benefits of sunlight: vitamin D deficiency might increase the risk of sudden unexpected death in epilepsy. Med Hypotheses, 74:158-61.

13. Sleeman, I., Aspray, T., Lawson, R., Coleman, S., Duncan, G., Khoo, T.K., Schoenmakers, I., Rochester, L., Burn, D., Yarnall, A. (2017). The Role of Vitamin D in Disease Progression in Early Parkinson's disease. J Parkinsons Dis, 7:669-675.

14. Larsson, S.C., Singleton, A.B., Nalls, M.A., Richards, J.B. (2017). International Parkinson's disease Genomics Consortium (IPDGC). No clear support for a role for vitamin D in Parkinson's disease: A Mendelian randomization study. Mov Disord, 32:1249-1252. 